Voix et Images

voixetimages

\title{
Avatars du biographique
}

\section{Robert Dion}

Volume 30, numéro 2 (89), hiver 2005

Les avatars du biographique

URI : https://id.erudit.org/iderudit/011240ar

DOI : https://doi.org/10.7202/011240ar

Aller au sommaire du numéro

\section{Éditeur(s)}

Université du Québec à Montréal

\section{ISSN}

0318-9201 (imprimé)

1705-933X (numérique)

Découvrir la revue

\section{Citer cet article}

Dion, R. (2005). Avatars du biographique. Voix et Images, 30(2), 17-20.

https://doi.org/10.7202/011240ar d'utilisation que vous pouvez consulter en ligne.

https://apropos.erudit.org/fr/usagers/politique-dutilisation/ 


\title{
AVATARS D U BIOGRAPHIQUE
}

\author{
$++$ \\ ROBERT DION \\ Université du Québec à Montréal
}

Le dossier de Voix et Images que voici se situe dans le prolongement d'une réflexion sur la biographie, et spécialement sur la biographie d'écrivain, menée en France depuis quelque temps et qui, jusqu'ici, a trouvé peu d'échos au Québec ${ }^{1}$. Ces dernières années, en effet, ont paru outre-Atlantique deux livraisons de la Revue des sciences humaines consacrées respectivement au «Biographique ${ }^{2}$ » et aux «Paradoxes du biographique $^{3}$ ", ainsi qu'un dossier de la revue Littérature ayant pour titre "Biographiques ${ }^{4} »$. Au Québec, à ce jour, rien de comparable n'a été publié sur la question; mais la sortie récente d'un tome des Archives des lettres canadiennes ${ }^{5}$ portant sur ce genre littéraire souvent mésestimé semble bien indiquer que quelque chose, ici, bouge sur le front de la biographie. Il s'agit sans doute de répondre à la "passion du biographique» qui s'est emparée des cercles savants après avoir comblé les attentes du grand public: depuis quelque temps, on ne compte plus les biographies érudites et les fictions biographiques destinées aux lecteurs du champ restreint. De tels ouvrages ont fini par imposer - et exposer - d'autres personnages que les vedettes médiatiques dont les «vies» colonisent les sections spécialisées des librairies et saturent l'espace public.

Toutefois, ce qui nous retiendra dans ces pages, c'est bien davantage le «biographique» que la biographie. Celui-ci se caractérise, pour reprendre le mot d'Alain Buisine, par le retour de «l'écrivain en personne» sous ses divers avatars, tant dans des fictions, des essais et des pièces de théâtre que dans des biographies proprement dites, dont les formes couvrent tout l'empan qui va de la biographie «classique» (André Maurois, Henri Troyat, etc.) jusqu'aux fictions biographiques les plus osées, dans la filiation des Vies imaginaires de Marcel Schwob ${ }^{6}$. «Ce qui m’apparaît

$$
++
$$

1 Depuis 2000, Frances Fortier et moi-même menons un projet de recherche sur les biographies fictives d'écrivains dans le cadre plus général d'une réflexion sur la dynamique des genres littéraires. $\mathbf{2}$ Revue des sciences humaines, $\mathrm{n}^{\circ}$ 224, «Le biographique», 1991. 3 Revue des sciences humaines, $\mathrm{n}^{\circ} 263$, "Paradoxes du biographique», 2001. 4 Littérature, n 128, «Biographiques», 2002. 5 Dominique Lafon, Rainier Grutman, Marcel Olscamp et Robert Vigneault (dir.), Approches de la biographie au Québec, Montréal, Fides, coll. "Archives des lettres canadiennes», t. XII, 2004. 6 Marcel Schwob, Vies imaginaires, Paris, Gallimard, coll. «L'imaginaire», 1998 [1896]. 
désormais décisif, c'est que le biographique n'est plus l'autre de la fiction", écrit Buisine, qui ajoute: «La biographie est elle-même devenue productrice de fictions, bien plus elle commence à comprendre que la fictionnalité fait nécessairement partie du geste biographique ${ }^{7} . »$

Au Québec aussi, le biographique a passé les frontières de la biographie stricto sensu, même si celle-ci a connu un important développement ces dernières années avec, par exemple, le lancement d'une collection comme «Les grandes figures» chez XYZ éditeur ou le succès des ouvrages de François Ricard sur Gabrielle Roy ${ }^{8}$, de Marcel Olscamp sur Jacques Ferron ${ }^{9}$ ou d'Hélène Pelletier-Baillargeon sur Olivar Asselin ${ }^{10}$. Cela dit, on ne trouvera pas dans le présent numéro une histoire ni un état présent du développement de la biographie au Québec, les Archives des lettres canadiennes s'étant chargées de cette tâche. Les collaborateurs s'attacheront plutôt à débusquer les extensions des formes (et usages) du biographique: car il semble que son inscription dans la littérature québécoise actuelle soit sujette à maintes stratégies de transposition et de détournement. C'est peut-être un signe de la méfiance que les traditions française et francophone entretiennent vis-à-vis de l'évocation biographique depuis au moins Mallarmé, Proust et Valéry et, plus radicalement encore, depuis la Nouvelle Critique et le Nouveau Roman. Or les œuvres dont il sera question dans le présent dossier, surtout fictionnelles mais aussi critiques, bravent toutes, d'une manière ou d'une autre, l'interdit du biographique, de la vie d'auteur et, ce faisant, inventent de nouvelles façons de penser le récit de vie et le rapport entre vie et œuvre. En cela, elles touchent à plusieurs enjeux fondamentaux de la réflexion théorique actuelle, comme le rapport au canon littéraire incarné par les écrivains biographés, la dimension (auto)biographique des fictions mettant en scène des écrivains et la légitimité du recours à l'information biographique dans le discours savant sur les textes.

En ouverture du dossier, Jean Morency mesure les conséquences de la pratique en parallèle, par Victor-Lévy Beaulieu, d'une écriture biographique et d'une écriture de fiction, montrant comment chez cet écrivain, à l'instar de ce qu'on observe chez Stefan Zweig par exemple, les deux versants du travail littéraire s'épaulent, mais aussi, de façon moins apparente, se cannibalisent. En Beaulieu, le romancier et le critique se rencontrent sur le terrain de la biographie, ce qui ne va pas sans générer des tensions auxquelles Morency est particulièrement attentif. La contribution suivante, signée par Catherine Dalpé, Mahigan Lepage et moi-même, traite du Triptyque des temps perdus de Jean Marcel, composé de trois "panneaux» mettant en scène les figures historiques d'Hypatie d'Alexandrie, de saint Jérôme et de Sidoine Apollinaire. Tenus de ne retenir qu'une fraction de la richesse de cet «ensemble»,

$$
+++
$$

7 Alain Buisine, «Biofictions», Revue des sciences humaines, n 224, 1991, p. 10. 8 François Ricard, Gabrielle Roy. Une vie, Montréal, Boréal, 1996. 9 Marcel Olscamp, Le fils du notaire, Jacques Ferron 1921-1949. Genèse intellectuelle d'un écrivain, Montréal, Fides, 1997. 10 Hélène Pelletier-Baillargeon, Olivar Asselin et son temps, t. I, Le militant, Montréal, Fides, 1996; t. II, Le volontaire, Montréal, Fides, 2001. 
nous avons choisi de l'aborder du point de vue de l'amalgame des genres - roman historique, roman biographique - , cherchant à indiquer de quelle manière le travail sur les codes génériques fait surgir, d'une forme censément «dépassée» et d'une histoire révolue, une modernité tant thématique que scripturale.

On lira ensuite l'article d'Élisabeth Nardout-Lafarge sur la présence de Réjean Ducharme dans quelques romans québécois récents. L'auteure y montre comment, de modèle littéraire pour des auteurs comme Louis Hamelin et Gaétan Soucy, le "grantécrivain ${ }^{11}$ " Ducharme devient un modèle au sens pictural chez Hugo Roy, Monique Proulx et Catherine Mavrikakis. Nardout-Lafarge est conduite à dégager les stratégies par lesquelles les trois romanciers tentent de s'approprier une figure désormais «classique» de la littérature québécoise, d'autant plus prégnante qu'elle est d'une certaine manière absente et qu'elle résiste à sa captation. Du coup, c'est l'œuvre qui devient le principal support du discours biographique - œuvre citée ou commentée, imitée, parodiée ou déployée fictionnellement. On se réjouira du fait que cette omniprésence d'un discours sur le texte ducharmien dans les fictions biographiques incite Nardout-Lafarge à mener une réflexion soutenue sur l'intertextualité qui apporte un éclairage inédit sur l'écriture biographique.

Praticien du genre de la biographie, Bernard Andrès nous introduit dans la «fabrique du biographique», pour ainsi dire, faisant valoir son rôle et ses pouvoirs non seulement dans le cadre du travail littéraire, mais aussi et surtout dans celui du travail savant. À partir de ses travaux sur Laterrière, mais en envisageant également une "prosopographie de l'aventurier» à l'époque du Régime français et de la Conquête, Andrès laisse entrevoir une histoire et une critique littéraires enrichies par l'apport de la biographie, que cette dernière se limite aux certitudes de la science ou qu'elle s'ouvre, plus ou moins timidement, aux lumières de la fiction. Enfin, en clôture du dossier, Frances Fortier, Caroline Dupont et Robin Servant s'attachent à «un biographique dramatisé par lequel il s'agit d'incarner des écrivains à la scène». Par l'analyse des pièces biographiques de Michel Garneau, Jovette Marchessault, Anne Legault, Robert Lalonde, Victor-Lévy Beaulieu et Ralph Burdman, les auteurs entendent faire surgir les phénomènes de transposition qui affectent le savoir biographique quand celui-ci se déporte de la narration vers la représentation - quand le dramaturge doit inventer les paroles prononcées par des personnages historiques, jusqu'à leur donner corps sur la scène.

Il ne reste, en terminant, qu'à souhaiter que les diverses contributions présentées dans ces pages permettent de mettre en lumière aussi bien les diverses incarnations du biographique dans la littérature québécoise récente que les enjeux divers - esthétiques, cognitifs, institutionnels, etc. - de sa prolifération actuelle.

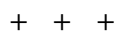

11 Le mot est de Dominique Noguez qui, avec Les trois Rimbaud par exemple, tente une certaine réhabilitation - quoique ludique et parodique - de la critique biographique. Dans «Ressusciter Rimbaud», il écrit: «[S]i j'ai choisi de ne pas limiter mes "pastiches par anticipation" aux textes, mais de les étendre en quelque sorte à la vie même, c'est que Rimbaud nous rappelle avec éclat l'importance de la vie, du biographique dans la création" (Jean Larose, Gilles Marcotte et Dominique Noguez, Rimbaud, Montréal, Hurtubise HMH, coll. «L'atelier des modernes», 1993, p. 121-122). 


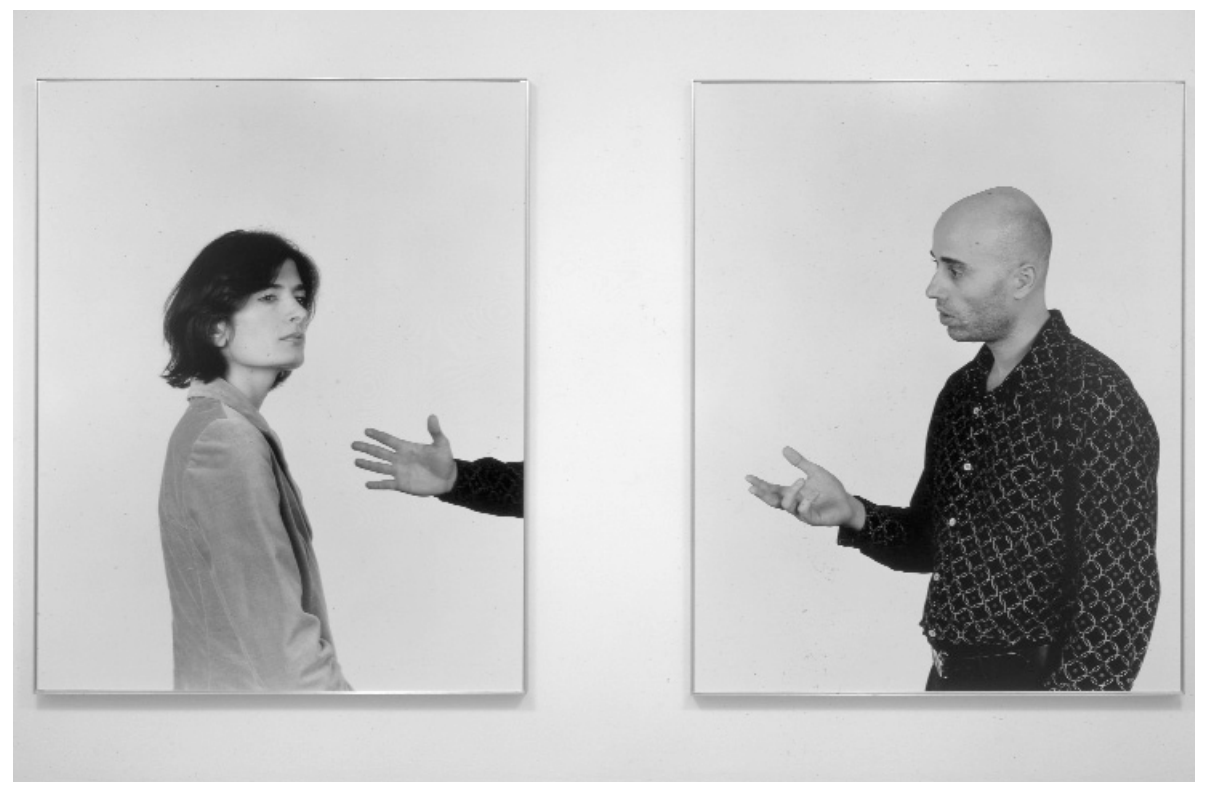

Geneviève Cadieux, Elle et Lui, 1997, diptyque, épreuve chromogène montée sur plexiglas, chaque élément: 197,5cm x 159 cm. Crédit photo: Richard-Max Tremblay. 